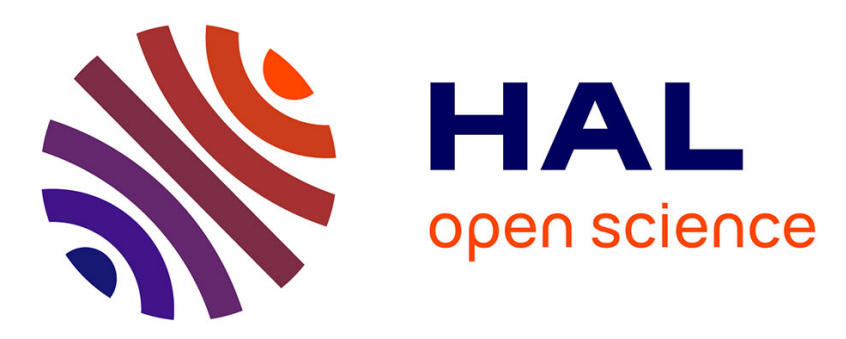

\title{
Exposer le miracle ou mettre de l'ordre dans le désordre de la foi
}

\author{
Caroline Perrée
}

\section{To cite this version:}

Caroline Perrée. Exposer le miracle ou mettre de l'ordre dans le désordre de la foi : Étude de trois salles votives au Brésil, en France et au Mexique. Terrains et Travaux: Revue de Sciences Sociales, 2017, Varia, 30. halshs-01565568

\section{HAL Id: halshs-01565568 https://shs.hal.science/halshs-01565568}

Submitted on 19 Jul 2017

HAL is a multi-disciplinary open access archive for the deposit and dissemination of scientific research documents, whether they are published or not. The documents may come from teaching and research institutions in France or abroad, or from public or private research centers.
L'archive ouverte pluridisciplinaire HAL, est destinée au dépôt et à la diffusion de documents scientifiques de niveau recherche, publiés ou non, émanant des établissements d'enseignement et de recherche français ou étrangers, des laboratoires publics ou privés. 


\section{Caroline Perrée}

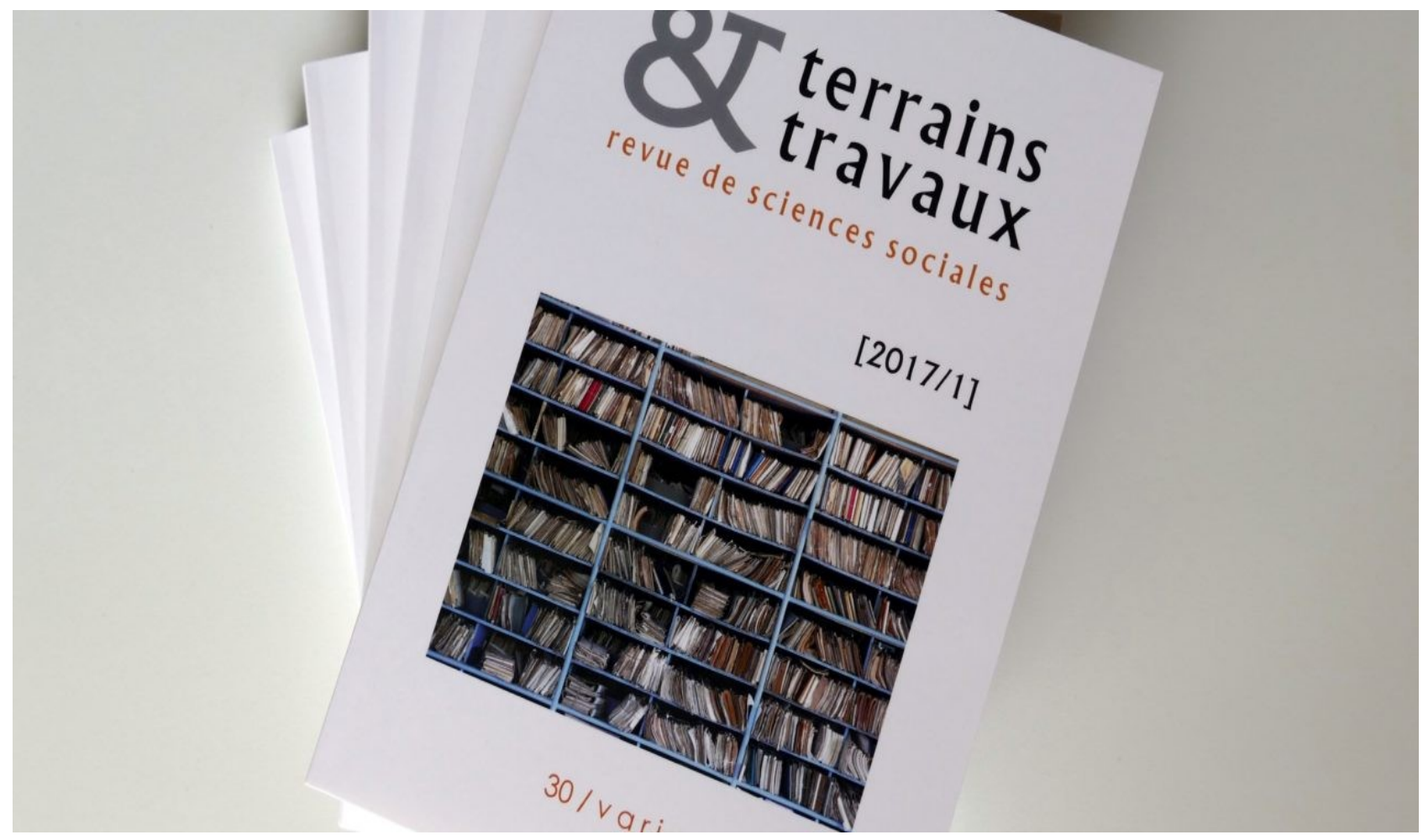

\section{Pour citer cet article :}

Perrée, C. (2017). Exposer le miracle ou mettre de l'ordre dans le désordre de la foi: Étude de trois salles votives au Brésil, en France et au Mexique. Terrains \& travaux, 30,(1), 143-162. http://www.cairn.info/revue-terrains-et-travaux-2017-1-page-143.htm.

\section{Résumé}

\section{Français}

L'existence de salles d'ex-voto au cœur de sanctuaires catholiques pose la question des raisons qui conduisent le clergé à exposer un objet non liturgique. L'examen au cours des quinze dernières années de trois salles de lieux de culte au Brésil, en Bretagne et au Mexique permet de repérer les différents dons votifs actuels mais surtout d'analyser les logiques sous-tendant leur exposition. Celle-ci varie d'un lieu de culte à l'autre et révèle des logiques de concession de la part de l'Église face aux formes que revêt la dévotion populaire, des logiques de mise en scène du miracle à des fins évangélisatrices et des logiques patrimoniales de muséification du miracle. Notre étude repose sur plusieurs travaux de terrain visant à décrire ces salles votives pour mieux cerner et comprendre les stratégies que recèle la monstration des ex-voto contemporains. 


\section{Mots-clés}

- ex-voto

- clergé

- patrimonialisation

- muséification

\section{English}

Displaying the Miracle, Ordering the FaithA Study of three ex voto rooms in Brazil, France and MexicoThe existence of rooms for the display of votive offerings inside Catholic sanctuaries questions the reasons for which the clergy puts these non-liturgical objects on display. Over the last fifteen years, we have analysed three rooms devoted to ex-voto in places of worship, in Brazil, Brittany and Mexico, in order to understand the variety of votive offerings as well as the logics underlying their display. These vary from site to site and reveal a logic of concession on the part of the Church to forms of popular devotion ; a logic of staging the miracle for evangelizing purposes ; and a patrimonial logic concerned with the museumification of the miracle. Our study is based on ethnographic fieldwork documenting these votive rooms to better identify and understand the strategies of demonstration of contemporary ex-votos.

\section{Keywords}

- ex-voto

- clergy

- national heritage

- museumification

\section{Plan de l'article}

1. Introduction

2. Préambule méthodologique

3. De la salle votive à la salle des ventes : la valeur utilitaire de l'ex-voto

4. La mise en scène du miracle : la valeur probatoire de l'ex-voto

5. Le miracle muséographié : l'ex-voto au service du patrimoine

6. Conclusion

\section{En ligne :}

http://www.cairn.info/revue-terrains-et-travaux-2017-1-p-143.htm

\section{Notice biographique :}

Caroline Perrée est chercheuse en Histoire de l'Art au CEMCA à Mexico (UMIFRE 16 CNRS). Ses recherches s'inscrivent dans le champ de l'anthropologie de l'image par l'analyse des pratiques et images religieuses et de leurs interactions avec les créations artistiques contemporaines, notamment au Mexique. Elle travaille plus particulièrement sur les usages religieux et esthétiques de l'objet votif depuis 2002. Ses recherches portent plus généralement sur les questions de temporalité en art. 


\section{Exposer le miracle ou mettre de l'ordre dans le désordre de la foi : étude de trois salles votives au Brésil, en France et au Mexique}

\section{Caroline Perrée}

\section{Introduction}

À l'instar de l'amulette ou de l'offrande, le mot « ex-voto» fait aussitôt surgir toute une kyrielle d'objets hétérogènes dont la destinée votive n'a rien d'évident. Parce que ce type d'objet est né de la dévotion populaire, nul dogme n'encadre ni la pratique ni la forme de l'ex-voto. Provenant de la contraction de la locution latine ex-voto suscepto qui signifie « d'après le vœu fait », l'ex-voto désigne tout objet offert pour un miracle demandé ou accompli. Dans la pratique c'est ce dernier, l'ex-voto gratulatoire, offert en remerciement d'un miracle, qui est le plus représenté (Cousin, 1979 : 108). Objets protéiformes depuis leurs origines les ex-voto contemporains se caractérisent aujourd'hui encore par leur hétérogénéité. Parce qu'elle se définit comme un pacte (Cousin, 1979 : 114), la pratique votive sous-tend l'offrande d'un don générant un contre-don, une promesse accomplie en échange du miracle accordé. En effet, l'ex-voto tel qu'il est utilisé depuis l'Antiquité entre dans la catégorie des « actes générateurs d'obligations » (Turlan, $1955: 515$ ), en même temps qu'il revêt une valeur probatoire de l'intervention divine puisqu'il atteste la réalisation du miracle obtenu (Sigal, 1985 : 90). C'est cette dimension probatoire que l'Église a choisie de privilégier en acceptant de l'exposer dès le Moyen Âge, et ce à des fins évangélisatrices, mais aussi pécuniaires, car l'exposition des ex-voto permet de favoriser le flux de pèlerins vers des sanctuaires réputés miraculeux (Sigal, 1985 : 91). Cependant, et malgré son extraordinaire survie au fil des siècles, l'origine «païenne» de l'ex-voto n'a cessé de le faire percevoir comme « une pratique non cléricale » (Cousin, 1979 : 115). Ainsi, l'exposition de l'ex-voto dans un lieu catholique ne va pas de soi et révèle aujourd'hui encore de la part de l'Église une attitude qui oscille entre la concession et l'intégration.

La double problématique inhérente à l'ex-voto - labilité formelle et pratique «païenne » présente dans les lieux de culte - est manifeste lorsque que l'on franchit le seuil d'une salle d'exvoto. Sous le contrôle de l'Église, cette salle illustre à la fois le caractère hétéroclite de l'ex-voto et les choix faits en matière d'exposition d'objets dévotionnels non liturgiques. Ainsi, l'Église par le tri qu'elle opère définit ce qui est votif de ce qui ne l'est, ces salles d'ex-voto sont alors des indicateurs de l'attitude du clergé à l'égard d'objets non catholiques, et soulèvent plusieurs questions : qu'est-ce qui caractérise l'objet exposé comme votif? Quels sont les intérêts d'un sanctuaire à exposer des objets non conformes au dogme ? Quels critères de sélection président alors à leur sélection et dans quels buts? 
Notre analyse porte sur trois salles d'ex-voto implantées dans des lieux de culte importants mais secondaires : Trindade au Brésil, San Juan de los Lagos au Mexique et Sainte-Anne d'Auray en France. Ce choix vise volontairement à travailler sur des sites moins connus que ceux de NotreDame d'Aparecida dans l'état de São Paolo, de la Basilique de Guadalupe à Mexico et de Lourdes en France, et qui sont particuliers dans leur fonctionnement mais comparables entre eux par la place qu'ils occupent dans la dévotion populaire et par l'affluence qu'ils génèrent. Parce que ces trois lieux géographiques sont soumis à une histoire et à un contexte socio-religieux différents, ils nous permettent d'analyser la variété des options prises par le clergé quant à l'existence et au rôle que peuvent tenir les salles d'ex-voto dans un lieu catholique. Et l'on peut alors déceler trois tendances générales selon la valeur attribuée à l'ex-voto : une valeur utilitaire, une valeur probatoire et une valeur patrimoniale. Si chaque lieu adopte une politique globale dans la gestion de l'ex-voto, une ethnographie des trois salles révèle que plusieurs valeurs sont à l'œuvre au sein d'un même sanctuaire. C'est pourquoi, nous analyserons ces salles à l'aune de la valeur attribuée à l'objet votif.

\section{Préambule méthodologique}

Notre étude s'intéresse plus particulièrement à trois salles précises au Brésil, en France et au Mexique, mais ce choix repose sur une fréquentation assidue des salles d'ex-voto notamment au Mexique depuis 2003, date à laquelle nous avons commencé à travailler sur l'objet votif. Cette fréquentation répondait au besoin de définir l'ex-voto, parce que les votifs se caractérisent par leur éclectisme sur le plan formel. L'on peut ainsi offrir des membres anatomiques fabriqués à cet effet, en cire, en métal ou en bois mais aussi des grenouillères pour remercier de la réussite d'une opération d'un enfant ou de sa guérison miraculeuse, des peluches, des cheveux, des robes de mariée, des béquilles, etc.

Objet témoin, il prend la forme que le dévot veut bien lui donner, mais il entretient un lien avec le miracle, le récipiendaire ou le mal enduré. Ce lien est figuratif dans le cas des ex-voto anatomiques et des peintures votives. Mais il est très souvent symbolique et ne permet pas toujours de comprendre pour quels maux ou en remerciement de quels miracles l'objet est offert. Il s'inscrit alors dans des logiques diverses, par exemple celles de contact et de substitution. Cette dernière était courante au Moyen Âge, où l'on offrait son poids en cire pour obtenir une guérison (Sigal, 1983 : 4, 19). Aujourd'hui, la tresse de cheveux au Mexique ou au Brésil peut aussi se substituer au donateur dans la demande ou le remerciement. Dès lors, un lieu nommé « salle d'ex-voto », ou sala de milagres au Brésil, authentifie l'objet entreposé comme votif, c'est-à-dire donné en échange d'un miracle. Ainsi, la variété des objets offerts explique la pluralité formelle de l'ex-voto, qui donne lieu

à l'établissement de nombreuses typologies (Bautier, Sigal, Boullet) permettant de mieux 
comprendre les logiques sous-jacentes au don votif.

Durant plus de quatre ans, de 2002 à 2006, nous avons visité une dizaine de salles d'ex-voto, le plus souvent au Mexique. Afin d'établir des typologies votives sur les plans formel et fonctionnel, nous avons travaillé sur d'autres pays comme la France et le Brésil à titre comparatif. Cette analyse se concentre particulièrement sur San Juan de los Lagos. En effet, c'est à la fois le pays et le sanctuaire que nous connaissons le mieux grâce à de fréquentes visites espacées dans le temps : en février 2003, en janvier 2014 et en novembre 2016. Ce dernier terrain d'observation a été un moment privilégié d'exploration de l'ensemble des lieux votifs, en compagnie du prêtre qui en a la charge, ainsi que l'interview de personnes laïques œuvrant dans ces espaces votifs. Quant au Brésil, notre visite à Trindade remonte à juillet 2004 et repose surtout sur une analyse de l'organisation de l'espace votif. L'observation à Sainte-Anne d'Auray en août 2004 a fait directement suite à Trindade dans une perspective comparatiste de la muséographie du miracle. Cette visite a été complétée par l'interview en septembre 2015 de Cécile Perrochon, qui est en charge des collections du sanctuaire de Sainte-Anne d'Auray, en tant que responsable du patrimoine.

D'une salle d'ex-voto à l'autre, des fonctionnements différents apparaissent selon les choix faits par les autorités cléricales car, derrière la spontanéité et le désordre apparents de certains lieux, c'est bel et bien le clergé qui est responsable de cette salle et de ce qui s'y dépose. Ce que nous avons sous les yeux est le résultat d'une volonté qui ressort de l'institution du sanctuaire. Nous en avons eu la confirmation à San Juan de los Lagos au Mexique lors de notre dernier travail de terrain en novembre 2016, lors d'une conversation avec l'homme chargé par le prêtre d'enlever les dons non accompagnés d'un écrit relatant le miracle, ainsi que les demandes que l'on ne peut pas faire à la Vierge, comme celle de la puissance sexuelle. À nos questions au prêtre sur l'ordre mis dans la salle, il a seulement répondu qu'il laissait les ex-voto les plus récents sans donner davantage de détails. Ce contrôle, voire cette censure, nous ont aussi été confirmés par Patricia Arias, spécialiste des ex-voto peints mexicains : les ex-voto évoquant la violence conjugale étaient rapidement éliminés par les prêtres et sacristains ${ }^{1}$. Ces ex-voto existent (Durand et Arias, 2002 : 103-106) mais par le passé ils n'étaient pas laissés dans la salle. À Trindade au Brésil, ce contrôle était évident car de grandes caisses en bois étaient installées pour faire le tri entre les dons.

Il n'est pas toujours simple d'obtenir des prêtres des informations quant à la gestion des salles d'ex-voto, car même quand il sont coopératifs, ils sont conscients qu'ils acceptent ce qu'ils nomment eux-mêmes une « offrande » en espagnol, ofrenda, qui n'a rien de catholique. Par ailleurs, comme ils se débarrassent de nombre d'entre elles, il est délicat pour eux d'aborder cet aspect. À Sainte-Anne d'Auray, nous n'avons pas eu ce problème car la personne en charge de la salle est une responsable du patrimoine, son discours et son attitude ne sont donc pas dictés par le dogme 
catholique. Nous privilégions un travail d'observation de l'organisation des salles d'ex-voto et des modalités d'exposition du miracle, et quand c'est possible, nous interviewons également les personnes en charge $\mathrm{du}$ bon fonctionnement des salles d'ex-voto. Cette ressource s'avère extrêmement utile pour comprendre les rouages de la gestion du votif, comme nous le verrons pour San Juan.

Pour mener à bien cette analyse d'une exposition d'un objet ni catholique ni esthétique issu de la dévotion populaire, nous aurons recours aux notions et concepts utilisés par Heinich dans un article rédigé suite à une enquête menée en 2004-2005 à la demande du service de l'Inventaire général (Heinich, 2009). L'enquête portait sur les critères choisis pour sélectionner les objets du patrimoine religieux issus de la dévotion ordinaire, qui ne sont pas des objets rares, critère incontournable de la logique patrimoniale. Or, les critères mis au jour recoupent ceux employés par l'Église de Trindade, de Sainte-Anne d'Auray et ceux de San Juan dans la partie muséographique, c'est pourquoi nous empruntons volontiers sa terminologie, d'autant que son article s'intéresse en particulier aux ex-voto et aux objets en série.

En effet, face à la pratique votive, le chercheur comme le prêtre se heurtent au problème du nombre et de la sérialité (Heinich, 2009 : 3). Un seul ex-voto de la série peut être conservé pour sa « représentativité », à savoir sa typicité, car il est emblématique de cette série et en représente la multiplicité. Mais, dans le même temps, un autre ex-voto peut aussi être exposé pour sa rareté, c'est-à-dire pour son caractère exceptionnel, et donc patrimonial, auquel s'ajoutent d'autres valeurs comme la beauté, l'ancienneté et l'authenticité (Heinich, 2009: 10). Ce sont ces valeurs qui président au choix de l'exposition des ex-voto dans nos trois salles mais à différentes échelles et à des fins patrimoniales ou probatoires.

\section{De la salle votive à la salle des ventes : la valeur utilitaire de l'ex-voto}

La ville de San Juan de los Lagos au Mexique est située dans l'état de Jalisco, dont la capitale est Guadalajara. C'est le deuxième lieu de pèlerinage après celui de la Vierge de Guadalupe. $\mathrm{Au} \mathrm{XVI}{ }^{\mathrm{e}}$ siècle, le frère Miguel de Bolonia pacifie le territoire et donne une image de l'Immaculée Conception à l'un des villages. Le premier lieu de culte prend la forme d'une petite chapelle en terre battue. L'image de la Vierge prend de l'importance à partir du XVII ${ }^{\mathrm{e}}$ suite au premier miracle intervenu en 1623 : la fille d'un voltigeur, morte suite à une chute, est ressuscitée après que la statue de la Vierge a été posée sur elle. La basilique actuelle date du XVIII ${ }^{\mathrm{e}}$ siècle, et les ex-voto conservés des $\mathrm{XIX}^{\mathrm{e}}$ et $\mathrm{XX}^{\mathrm{e}}$ siècles. Les peintures votives comprises entre 1840 et 1945 ont fait l'objet d'une étude précise par Marianne Bélard (Bélard, 1997).

Même si la salle votive existe bel et bien au sein de la basilique, elle s'avère petite en regard 
de l'affluence, de l'ancienneté du culte et des dons. Notre première visite remonte à février 2003 et la salle comportait plus ou moins le même nombre d'objets. Pourtant, le pèlerinage à San Juan est à la fois national et international puisque nombre de Mexicains vivant aux États-Unis continuent à venir régulièrement. L'explication nous est donnée lorsque nous découvrons que plusieurs lieux sont consacrés aux ex-voto : la salle de dépôt, le hangar de revente, l'entrepôt d'ex-voto peints, le musée privé et le musée public.

La salle, où l'on vient remettre son ex-voto, est située au fond du sanctuaire. C'est une sorte d'antichambre qui mène au «Camarín » (la Chambre), la pièce où l'on habille la Vierge de ses plus beaux atours. La montée de l'escalier se fait au milieu d'ex-voto divers accrochés à des parois aménagées à cet effet par le clergé, car elles sont recouvertes de panneaux perforés servant habituellement à poser des outils. Sur ceux-ci sont suspendus des photos de différents formats, des dessins illustrés par une légende, des papiers écrits, des tresses de cheveux, quelques vêtements d'enfants, curieusement fort rares en comparaison d'autres salles d'ex-voto mexicaines. Des cadres ou des boites en verre sont également installés, sur lesquels de petites pancartes indiquent que seul le don de cheveux est autorisé, mais les gens accrochent aussi des milagritos $^{2}$ dans les cadres. Sont également posées sur les marches quatre ou cinq bicyclettes sans autre forme d'explication.

On distingue encore de-ci de-là quelques ex-voto peints récents de 2006, 2007, 2010 et de 2013. Mais ces petites peintures sont de plus en plus remplacées par des ex-voto narrant le miracle de manière plus composite, à savoir des ex-voto créés par le donateur lui-même sous la forme d'un collage mêlant photo, dessin, image pieuse de la Vierge collée sur le support votif, et accompagnés d'un écrit expliquant les circonstances du don. En revanche, en hauteur et non accessibles sont exposés des dizaines d'ex-voto peints plus anciens mais placés trop haut pour pouvoir être lus ou simplement observés, afin d'éviter qu'ils ne soient pas volés par des collectionneurs.

La plupart des remerciements concernent une opération chirurgicale, comme l'attestent les collages narratifs ou les photos de personnes opérées. Nombre de demandes de protection émanent de femmes enceintes mais aussi de mères pour la protection de leurs enfants en bas âge car la Vierge de San Juan est très sollicitée par les mères. Les fautes d'orthographe ponctuant les écrits témoignent de l'humilité des pèlerins. Quant aux milagritos, ils sont disposés ça et là figurant le suppliant en prière ou le membre à guérir ; ceux représentant des animaux rappellent l'importance de l'élevage dans l'état de Jalisco et le milieu rural dont sont issus les donateurs. Les tresses, quant à elles, constituent un ex-voto récurrent à San Juan de los Lagos, parfaitement assimilé par l'Église comme l'indique la pancarte en autorisant le dépôt. Parmi les dons, il y aussi de l'argent notamment des dollars offerts par des Mexicains vivant aux États-Unis, qui sont donnés directement à l’Église,

2 Les milagritos, que l'on peut traduire par « petits miracles », sont des ex-voto anatomiques en métal de petite taille, à savoir moins de $2 \mathrm{~cm}$ en général. Ils sont très usités dans la pratique votive et sont accrochés le plus souvent directement sur la robe des saints dans les églises. 
comme nous l'explique une dame venue apporter les 25 dollars que sa belle-mère avait promis à la Vierge. Cette femme est morte mais sa belle-fille vient faire ce don votif post-mortem, ce qui souligne l'importance accordée à la promesse dans la démarche votive ${ }^{3}$.

L'accrochage dans la salle est spontané et immédiat. Les pèlerins entrent, regardent les exvoto, lisent les miracles attentivement, les commentent et suspendent leurs ex-voto avant de sortir. Malgré cette apparente liberté, la salle reste sous contrôle car un homme d'entretien est chargé d'ôter certains ex-voto. Lors de notre visite en janvier 2014, il retirait plus volontiers les vêtements, les milagritos et les sacs en plastique volumineux contenant des cheveux. Il procédait en pleine journée enlevant parfois les ex-voto à peine accrochés sans qu'aucun donateur ne réagisse. Lors de notre visite en 2016, c'était une autre personne qui enlevait les dons en soirée et plutôt en semaine, à des moments où l'affluence est moindre. Comme nous l'explique le prêtre, certains dévots tiennent à ce que leurs dons restent dans la salle et viennent le vérifier le temps de leur séjour. C'est pourquoi le prêtre demande à ce qu'on les laisse quelques jours, puis ils sont remisés au « Bazar ». En effet, nous ne sommes pas au bout de nos surprises lorsque nous découvrons que les dons votifs retirés sont intégrés à un processus de vente orchestré par les autorités ecclésiastiques.

Près du puits où se trouve la chapelle du Pozito du premier miracle, au milieu du dédale des marchands ambulants à 500 mètres de la cathédrale, l'on trouve un hangar immense peu remarquable de l'extérieur car il n'est désigné par aucune pancarte, mais il regroupe un nombre considérable d'objets mis à la vente : il s'agit des objets votifs exposés dans la salle précédente. Trois femmes laïques s'occupent du « Bazar», comme on le nomme, et entreposent les objets par catégorie : des grilles entières couvertes de tresses, chacune vendue à 20 ou 30 pesos (un peu plus de un euro), des dizaines de grenouillères pour bébés et des tee-shirts pour enfants sont suspendus sur des portants et vendus 10 pesos (50 centimes d'euros). Des milagritos sont également en vente pour 3 pesos, alors que les marchands alentours les vendent en moyenne 10 pesos. Le lieu est donc très bon marché. Tout un côté de la salle est occupé par des dizaines de robes de mariée à vendre elles-aussi, dans un coin des cadres et des images pieuses; au milieu de la salle des bouquets de fleurs ayant servi à orner la basilique. Des centaines de dons sont remisés là en attendant de trouver preneurs.

La présence du prêtre nous a permis de nous entretenir avec ces femmes, qui étaient plus réticentes à répondre à nos questions en janvier 2014. Ainsi, l'une d'elles nous explique que l'Église revend les objets offerts à la Vierge pour aider les pèlerins détroussés par des pickpockets, lorsqu'ils viennent à la Basilique. L'argent sert également aux migrants d'Amérique Centrale, qui passent par San Juan, pour qu'ils puissent continuer leur voyage vers les États-Unis. Ainsi, la salle d'ex-voto se transforme en salle des ventes après le don, pour financer les plus démunis. Les acheteurs potentiels 
ne savent pas d'où viennent les objets vendus. Si on lui pose la question, notre informatrice leur dit que ce sont des ex-voto offerts à la Vierge de San Juan. Dans ce cas, nombre d'entre eux ne les achètent pas car ils ignorent si les circonstances du don furent heureuses ou malheureuses. L'objet votif est donc considéré comme "chargé » par les dévots. Quant aux cheveux, le prêtre nous explique que ce sont des coiffeurs qui viennent les acheter pour faire des perruques ou des extensions avec des cheveux naturels. Les objets les plus vendus sont les grenouillères, puis les robes de mariée. Il est fort probable qu'à Trindade la même stratégie de recyclage soit à l'œuvre tant l'afflux de dons est considérable, mais nous n'avons pas pu le vérifier. Dans le sanctuaire du Seigneur de Bomfim dans l'état de Bahia, les vêtements votifs sont rapidement donnés, et l'on dit que les cheveux offerts sont vendus pour faire des perruques (Cândido, 2016 : 121-122). Si l'information n'a pas pu être vérifiée, c'est que le clergé n'a pas mis en place un lieu de revente officiel, à la différence de San Juan.

La salle d'ex-voto de San Juan révèle une attitude de concession réciproque : en considérant le peu d'objets présents, les donateurs doivent se douter que leurs ex-voto ne sont là que provisoirement mais ils l'acceptent puisqu'on leur permet de suspendre leur objet. L'essentiel est de remplir sa promesse en apportant le don une fois que le vœu a été exaucé, ce qui correspond bien à la démarche votive. Le cas de l'argent offert post-mortem est à cet égard significatif. Le clergé, quant à lui, tolère de manière éphémère les objets les plus triviaux par un système de revente qui alimente ses bonnes œuvres. Seuls les objets témoignant d'une intervention catholique explicite sont conservés et exposés avec une valeur probatoire. Mais l'exposition recèle aussi une valeur morale, car le prêtre exige que soient retirées les demandes indécentes, comme la puissance sexuelle. En somme, aucune tentation du spectaculaire ni de l'historique n'orchestre la mise en scène de la salle des dons qui, de tous les lieux votifs du site, est celui qui est le plus visité. Le clergé de San Juan adopte une attitude clairement pragmatique au sujet des ex-voto, sans exploiter leur dimension miraculeuse à des fins spectaculaires. La majeure partie du temps, l'ex-voto ne fait que transiter par la salle de dépôt pour prendre place dans l'un des quatre autres lieux votifs qu'abrite le sanctuaire.

\section{La mise en scène du miracle : la valeur probatoire de l'ex-voto}

La ville de Trindade se situe dans l'état de Goiânia. Cet état rural vit essentiellement de l'élevage de bovins. Si sa capitale officielle est Goiás, Trindade est considérée, elle, comme la capitale de la foi. La première chapelle a été construite en 1843 et, en 1912 est inauguré le premier sanctuaire du Père Divin Éternel (Reinato, 2009 : 318). Le sanctuaire tel que nous l'étudions date de 1943 mais sa fréquentation grandissant, un nouveau sanctuaire a été construit à partir de 2012. 
L'histoire du culte commence aux environs de 1840 lors de découverte par le couple Constantino Xavier et Ana Rosa de Oliveira d'un médaillon en argile représentant la Sainte Trinité couronnant la Vierge Marie. À partir de cette découverte intervient toute une série de miracles à l'origine du culte. Ce sont les missionnaires rédempteurs qui ont régularisé le culte du Père Divin Éternel. Ce dernier est le troisième lieu de pèlerinage le plus important du pays.

Ce sanctuaire spectaculaire ${ }^{4}$ recèle une immense salle d'ex-voto, qui se révèle être un superbe musée votif. Loin du bric-à-brac attendu, la Basilique du Divino Padre Eterno, «Père Divin Éternel » fait régner l'ordre dans le monde du miracle. Sous l'édifice moderne, à peine soixante dix ans, s'étend la sala de milagres, espace immense compartimenté en fonction des exvoto exposés. À l'entrée, des listes de noms dactylographiés s'étalent sur les murs, sans autre forme d'explication. Plus loin, au milieu de la pièce, des centaines de photos rangées dans des portes coulissantes que chacun peut regarder. Impossible de glisser la photo qu'on a apportée : les vitrines sont soigneusement fermées. Derrière ces photos, un mur de peintures votives anciennes et récentes, comprises entre 1945 et 1985, présente des miracles en couleur. Ces ex-voto sont très souvent anonymes et se caractérisent par une simple peinture du miracle sans forcément représenter le saint invoqué ou remercié. L'ensemble constitue une immense source visuelle pour l'historien et remplace le document écrit (Reinato, 2009: 320). Dans ce même espace, où des centaines de gens déambulent, une partie est également réservée aux objets orthopédiques, corsets, ainsi qu'aux exvoto anatomiques, anciens semble-t-il car ils sont en bois, petits ou grandeur nature. En s'enfonçant dans la salle, une autre partie s'ouvre aux visiteurs sur les objets de la ferme : charrettes, outils agricoles, récipients, etc. ; une autre expose des trophées, peaux de serpents et de crocodiles, et sur un mur sont accrochées des armes anciennes. Enfin, au fond de la pièce sont alignées quatre grandes caisses en bois portant des étiquettes, à gauche « Photos », à droite « Objets ». C'est ici, après avoir eu toutes les preuves matérielles des miracles accomplis, que le pèlerin dépose l'ex-voto qu'il est venu apporter.

Le lieu s'apparente à un musée car il est organisé à partir de thématiques votives et recèle une dimension de conservation et d'exposition d'un patrimoine votif. Le lieu ainsi orchestré dresse une véritable nomenclature des typologies votives, même si au Brésil leur infinité rend impossible un catalogage quantitatif, tandis qu'un catalogage typologique s'avère difficile puisque chaque nouvel ex-voto apporté peut remettre en question les catégories existantes (Oliveira, 2011 : 13). Mais aucun écrit ne vient entériner cette fonction muséale. En effet, les objets les plus étonnants sont soigneusement mis en scène sans la moindre explication sur les circonstances du don : remerciements, demandes de protection ou dangers encourus ? Le pèlerin ne peut que constater l'existence du miracle par l'objet déposé sans en connaître la teneur faute d'explication écrite. En

4 Notre étude date d'août 2004 et porte donc sur le sanctuaire construit en 1943. 
réalité, à Trindade l'ex-voto se donne à voir comme un véritable spectacle. L'ensemble est bien trop ordonné et ne permet aucune initiative personnelle, cet agencement « sous contrôle » ecclésiastique des ex-voto ôte toute spontanéité au don. En effet, le dépôt se fait à la fin de la visite dans ces caisses en bois que l'Église se chargera de trier et d'exposer mais pas a priori, dans la sala de milagres car celle-ci ne contient que des objets anciens et imposants. Et à voir l'aspect monumental des ex-voto, c'est bien le critère de rareté, défini par Heinich comme la recherche du «monumental» au sens de l'exceptionnel (2009: 10), qui est retenu par le clergé car sont privilégiés les dons les plus imposants, précieux, étonnants et rares.

De fait, le clergé de Trindade privilégie à la fois le "régime de singularité" - ce qui est insubstituable -, comme le sont les collections de bêtes exotiques, et "le régime de communauté" ce qui est partagé - (Heinich, 2009 : 5) comme les photos, tout en faisant une très large part au monumental, dont parle Heinich à propos du critère de rareté, et qui n'a rien d'une métaphore dans la cas de Trindade. L'immensité même de la salle rentre dans cette logique du démesuré. Les dons rares et monumentaux trouvent une place de choix et sollicitent toute l'attention des spectateurs et leur incrédulité. On pouvait voir en 2004 une voiture présentée à la curiosité des fidèles. Un papier collé sur le pare-brise expliquait qu'elle avait été volée en l'an 2000. Son propriétaire avait alors promis au Père Divin Éternel de la lui offrir en gage de remerciement s'il la retrouvait. Sa demande a été entendue et, quand il a eu l'argent nécessaire, il en a acheté une autre et a offert en ex-voto celle qui lui avait été volée (Perrée, 2007 : 110). Évidemment, l'histoire est édifiante et le miracle engage le fidèle à offrir des objets tout aussi impressionnants, puisque le don doit être à la hauteur du miracle accompli.

Il n'y a pas forcément à Trindade une logique patrimoniale reposant sur l'ancienneté et la rareté de l'objet, qui présiderait au choix des ex-voto exposés. Même si ce critère de rareté existe bel et bien, il s'agit surtout d'impressionner les visiteurs par l'accumulation des dons. Que ceux-ci soient sériels, à l'instar des ex-voto anatomiques ou les pans entiers de photos ; qu'ils soient rares , comme les collections de peaux de bêtes tuées ; patrimoniaux telles que les collections d'armes anciennes, ou bien monumentaux comme la voiture, l'ex-voto est exposé pour saturer l'espace visuel. Son amoncellement manifeste une abondance du miracle. Cette monstration du miracle s'explique par le fait que la concurrence entre les religions est très forte au Brésil, car le catholicisme doit faire face aux Églises scientistes, évangéliques, pentecôtistes et autres qui rencontrent un vif succès dans le pays. Par cette exposition de l'ex-voto, le sanctuaire cherche à attirer des fidèles, en donnant à voir une valeur probatoire de l'intervention divine puisqu'il atteste la réalisation du miracle obtenu, comme c'était déjà le cas au Moyen Âge (Sigal, 1985 : 90).

La sala de milagres se révèle, donc, comme un lieu de surenchère et la pratique votive est exhibée à Trindade pour favoriser l'expansion de la foi. Finalement, l'ex-voto, loin d'être méprisé, 
devient l'un des instruments de la propagande catholique. Il se livre ici une véritable « guerre des images » (Gruzinski, 1999), qui sert de catéchèse pour mieux contrôler l'imaginaire des visiteurs (Reinato, 2009 : 329). Cette mise en scène n'est pas nouvelle, Anne Lepoittevin étudie l'exemple d'une église italienne à Curtatone en 1520 prenant en charge l'organisation des dons votifs pour orner le sanctuaire et développer son rayonnement (Lepoittevin, 2014). Mais elle avait aussi cours dès l'Antiquité notamment à Délos où l'offrande, du IV siècle avant Jésus-Christ au ${ }^{\text {er }}$ siècle après, était un élément théâtral mis en scène tant dans les temples que dans les catalogues par la syntaxe expérimentale la décrivant (Prêtre, 2014). Trindade perpétue donc une pratique qui a fait ses preuves pour attirer les foules, en ne laissant rien au hasard. La catégorisation y est extrême permettant par là même à l’Église de mettre de l'ordre dans le désordre de la foi.

\section{Le miracle muséographié : l'ex-voto au service du patrimoine}

Déambulant dans le hangar du «Bazar» de San Juan de los Lagos, quelle n'est pas notre surprise de constater que le haut des parois est couvert d'ex-voto peints. Placés très haut, ils ne peuvent être lus et il faut une autorisation du prêtre pour y avoir accès. Nul pèlerin en ce lieu, seuls des spécialistes y entrent, accompagnés par un prêtre. Les ex-voto sont regroupés en fonction de leur style pictural, c'est-à-dire selon des critères esthétiques. Ce sont surtout des ex-voto d'action de grâces assez simples et peu narratifs sur le plan pictural. Il sont majoritairement datés du XX $\mathrm{XX}^{\mathrm{e}}$ siècle. Nombreux sont ceux qui s'inscrivent dans une production en série. Pourtant, d'autres témoignent de véritables qualités artistiques et sont en bon état de conservation. Ce sont ceux-là que le prêtre choisit pour les exposer dans une salle privée située au-dessus de la Vierge dans la cathédrale, ainsi que dans le musée dédié à l'histoire religieuse du sanctuaire ouvert au public.

La salle privée est entièrement dédiée à la présentation d'une trentaine d'ex-voto des XIX ${ }^{\mathrm{e}}$ et $\mathrm{XX}^{\mathrm{e}}$ siècles d'une belle exécution, accrochés aux parois comme des tableaux dans un musée. Dans quel but sont présentés ces ex-voto dans un endroit réservé au clergé ? Nulle valeur probatoire n'est en jeu dans ce lieu. D'ailleurs, lorsqu'il nous les présente, le prêtre ne commente que leurs qualités esthétiques, auxquelles il semble particulièrement sensible, puisqu'il a consacré un ouvrage à l'histoire de la basilique et à ses miracles abondamment illustré par des ex-voto peints (Gutiérrez, 2016). C'est également lui qui assure les visites relatives à l'architecture et à l'art de la basilique. À l'évidence, c'est le critère de rareté, qui a présidé au choix du prêtre puisque, comme le rappelle Heinich (2009 : 10), l'objet sélectionné se singularise parce que sa rareté est associée à d'autres valeurs comme celles de la beauté, l'ancienneté et l'authenticité. Or, les ex-voto exposés témoignent de l'ensemble de ces valeurs : leurs qualités picturales sont indéniables ainsi que leur ancienneté et leur authenticité. La rareté est un critère de la logique patrimoniale (Heinich, 2009 : 9), qui permet 
la conservation et l'exposition des objets sélectionnés. Pourtant, ces ex-voto ne sont pas placés dans le musée au vu et et à la connaissance de tous, alors que celui-ci est géré également par le prêtre. Ils constituent une sélection privée exposée à des fins esthétiques et patrimoniales réservée aux religieux et à leurs invités. Il s'agit aussi sans doute de les protéger des vols, le sanctuaire de San Juan ayant été l'un des plus pillés sur le plan votif(Luke, 2012 : 126).

À San Juan de Los Lagos, un musée inauguré en 2004 (Luke, 2012 : 124) est dédié à la Vierge et abrite des ex-voto. Il se trouve au fond d'une boutique et il faut connaître son existence pour s'y rendre. Il prend la forme d'un long couloir constitué de 21 vitrines de taille moyenne. Chacune abrite des objets liturgiques et retrace l'histoire du culte de la Vierge de San Juan grâce à une muséographie qui mêle faits historiques et patrimoine religieux, en intégrant sa dimension miraculeuse, puisque deux vitrines sont réservées aux ex-voto. L'organisation muséographique de l'une d'elles est orchestrée par le prêtre, et se fait à partir du « régime de singularité »- ce qui est insubstituable - et de leur typicité (Heinich, 2009 : 9, 11). Sont en effet présentés des objets rares, étonnants et beaux : le couteau et les balles d'un pêcheur repenti, des œuvres créées par des artisans et témoignant d'un véritable savoir-faire, des minéraux précieux, etc. Mais l'on trouve également des ex-voto choisis pour leur typicité car ils représentent une série entière. Ainsi, le prêtre a décliné une série de milagritos en choisissant des jambes, de la plus précieuse en argent à la plus petite et courante. Il fait la même chose avec les cœurs, créant de véritables nomenclatures à partir d'objets sériels choisis pour leur typicité mais aussi leur rareté - les jambes en argent sont plus anciennes - et les dispose de manière esthétique, du plus grand au plus petit. De même, les ex-voto peints exposés se caractérisent par leurs qualités d'exécution et, comme la dédicace du miracle est trop loin pour être lisible, c'est la peinture qui prime. L'autre vitrine réunit elle aussi des ex-voto peints sélectionnés pour leurs qualités picturales. L'ensemble est organisé dans un souci didactique digne d'une logique muséale car l'écrit est bel et bien présent. Sur la vitrine, un texte explique ce qu'est un ex-voto, alors que le mot est rarement employé au Mexique car l'on préfère le mot retablo pour les ex-voto peints et ofrenda pour les objets, et ce même texte mentionne ses origines antiques. Nombre d'objets sont aussi accompagnées d'une étiquette expliquant les circonstances ayant motivé le don. La vitrine à l'image du musée réunit donc plusieurs valeurs : probatoires, historiques et esthétiques.

En France, si une tendance générale souligne un recul certain du don d'ex-voto, certaines régions s'affirment au contraire comme de véritables terres votives, notamment les régions côtières. Les ex-voto marins constituent l'une des plus anciennes catégories votives puisque, face aux risques maritimes, les marins phéniciens et grecs offraient déjà des statues et des peintures votives aux divinités de la mer dans la crainte des dangers à affronter lors des traversées (Boullet, $1978: 15$ ). Les côtes françaises ont vu fleurir maintes chapelles et églises votives, c'est le cas de Notre-Damede-la-Garde à Marseille ou de Sainte-Anne d'Auray en Bretagne. La Bretagne a pérennisé des 
pratiques traditionnelles souvent liées aux périls maritimes. Aujourd'hui, les dons votifs subsistent sous la forme de maquettes de bateaux, suspendus dans maints sanctuaires. Dès lors, on ne peut s'étonner d'entendre un prêtre déclarer que les fidèles sortiront les ex-voto de la chapelle pour les porter lors de la procession du Pardon ${ }^{5}$. Les églises bretonnes sont rarement investies d'objets issus du quotidien sans doute parce que des lieux de culte spécifiques sont institués à cet effet.

C'est notamment le cas de la basilique de Sainte-Anne d'Auray qui abrite, dans la salle dite $\mathrm{du}$ «Trésor», les ex-voto qui lui sont offerts car le lieu a une longue histoire votive. Au XVII ${ }^{\mathrm{e}}$ siècle, ce n'est qu'un hameau d'une cinquantaine d'habitants nommé Keranna. C'est un homme de trente ans, Yves Nikolazic connu pour sa piété et sa justesse, qui reçoit les premières manifestations de sainte Anne puis qui est le témoin de ses apparitions à partir du mois d'août 1623. Le pèlerinage commence en 1625 mais prend toute son ampleur avec la conversion du bandit mécréant Pierre de Keriolet qui, touché par la grâce de sainte Anne, devient aumônier des pauvres jusqu'à la fin de sa vie.

La basilique et la statue actuelle de sainte Anne datent du XVIII ${ }^{\mathrm{e}}$ siècle. Quant à la salle d'ex-voto, elle témoigne de l'histoire du culte et de sa renommée puisque des ex-voto offerts par des personnalités historiques y sont présentés, comme l'ensemble avec la chasuble donné par Anne d'Autriche en remerciement de la naissance de Louis XIV (Huchet, 2005 : 113). En hauteur, sont accrochés ici aussi les ex-voto peints, pour la plupart anciens. À l'entrée, des objets précieux, au fond les vêtements d'enfants les plus divers pliés avec soin et offerts le plus souvent en remerciement pour une naissance longtemps attendue. Puis, les objets avalés par des enfants comme des épingles ou de petits jouets rejetés au bout de quelques jours. Les étiquettes les accompagnant nous apprennent les circonstances de l'accident, quelques-uns laissent même la radiographie sur laquelle apparaît l'objet avalé. Un peu plus loin, plusieurs vitrines de bijoux occupent l'essentiel de la pièce, c'est l'un des ex-voto les plus répandus: alliance en remerciement d'un mariage, gourmette en argent pour avoir trouvé un emploi, etc.

Dans une autre vitrine, ce sont les bouquets et les chapeaux de mariée qui sont offerts en remerciement d'un mariage réussi. À côté des objets témoignant d'un succès sportif : médailles et maillots de cycliste. Entre deux vitrines, trône une relique en or, don précieux de quelque prélat. Une autre vitrine regroupe des objets militaires, une autre nous raconte le quotidien des prisonniers de la Seconde Guerre Mondiale venus apporter cartes, maquettes du camp, cannes qu'ils ont sculptées en prison en guise de remerciement, pour être sortis sains et saufs. D'autres regroupent des objets exotiques, masques, coquillages et outils. Les objets les plus précieux côtoient les plus humbles dans l'unique but de remercier sainte Anne. Du stylo aux bijoux anciens, le donateur offre ce qu'il a de plus cher sans que le lien entre l'objet donné et le problème vécu soit évident.

5 Interview de prêtre, août 2004, Morbihan 
À Sainte-Anne d'Auray, nous voici en effet au cœur d'un véritable musée votif à l'usage du croyant ou du simple visiteur, puisque la salle du Trésor est organisée pour satisfaire la foi du dévot et la curiosité du touriste. Celle-ci est d'ailleurs sous le contrôle d'une responsable du patrimoine en charge de la conservation des ex-voto, qui a accepté de répondre à nos questions au sujet de l'organisation et du fonctionnement de la salle d'ex-voto ${ }^{6}$. Son travail a pris la suite de celui d'une sœur qui durant une grande partie du $\mathrm{XX}^{\mathrm{e}}$ siècle a entrepris de consigner les circonstances du don d'ex-voto à sainte Anne. Le récit de miracle est un genre ancien en Occident mais sa consignation est déjà une manière d'exercer une fonction de « censeur » (Crémoux, $2001:$ 39, 44) en vérifiant sa véracité, ce qui permettait déjà d'encadrer les dons.

À la différence de Trindade et de San Juan, tous les objets offerts sont présentés dans la salle du Trésor. Le caractère muséographique de cette dernière s'impose d'emblée parce qu'elle est composée de vitrines dans lesquelles sont rangés les dons par catégories, et accompagnés d'une étiquette explicative comme dans un musée d'ethnologie. Nommée la « Salle du Trésor », la pièce regroupant les ex-voto est volontairement désignée comme un lieu qui donne une valeur aux objets présentés ; cette valeur est à la fois réelle dans le cas des objets historiques ou en matière précieuse, et symbolique en ce qui concerne les menus objets du quotidien. La salle est d'ailleurs la proie de nombreux vols, le plus souvent des objets en or, au point que les objets les plus précieux sur le plan pécuniaire sont mis au coffre.

Les objets historiques confèrent une valeur patrimoniale indéniable à la salle ainsi constituée tout en soulignant la puissance de sainte Anne. En effet, la chasuble donnée par Anne d'Autriche, les objets en or ou les objets rapportés par les soldats participent de cette création d'une salle votive patrimoniale. De la même façon, l'étiquetage des objets par un carton expliquant les circonstances $\mathrm{du}$ don renforce l'inscription de l'objet dans un contexte historique et miraculeux. Comme dans un musée, l'objet est en effet expliqué dans son contexte temporel et ancré dans une histoire nationale commune, tandis que le visiteur est invité à faire une contribution volontaire après avoir visité la salle.

Dès lors, à Sainte-Anne d'Auray l'ex-voto est intégré dans un patrimoine religieux, faisant clairement passer l'objet ordinaire de la dévotion populaire au statut d'objet historique par un processus de patrimonialisation. Ainsi, les objets fabriqués par les prisonniers de guerre sont regroupés pour mieux illustrer un événement historique national auquel un visiteur européen, croyant ou non, peut reconnaître une part de sa propre histoire : la Grande Guerre et la Seconde Guerre Mondiale. Dans son article, Heinich cite les propos de Pierre Nora remarquant que « le « régime de singularité » (valorisant ce qui est insubstituable) est indissociable de l'intérêt pour le patrimoine, même si celui-ci a également à voir [...] avec le « régime de communauté » (valorisant 6 Interview le 3 septembre 2015 de Cécile Perrochon, responsable du patrimoine de l'Académie de Musique d'Arts Sacrés. 
ce qui est partagé), en tant qu'il relève de l'intérêt général, de la nation, des lieux de mémoire » (Heinich, 2009: 9). Mis sous vitrine et étiqueté, l'objet votif témoigne à la fois du miracle individuel et de l'histoire patrimoniale commune.

Quant aux objets sériels, comme les vêtements d'enfants, les montres, les bijoux, les robes et les bouquets de mariée, ils sont mis sous vitrine en témoignage d'une histoire de la dévotion populaire, comme le serait un objet rituel africain dans un musée d'ethnologie. Cette sérialité si difficile pour effectuer des choix « devient un critère positif, parce qu'elle ouvre la possibilité d'une catégorisation et, avec elle, d'une interprétation, d'une attribution de sens, d'un discours sur la signification historique de l'objet en tant qu'élément de la typologie dans laquelle il s'insère » (Heinich, 2009 : 6). Le parallèle avec le travail des chercheurs sur la dévotion populaire révèle bien le processus de patrimonialisation auquel est soumis l'objet votif. Cette vision historique de l'exvoto est renforcée par le fait que c'est bel et bien une spécialiste du patrimoine qui est chargée de s'occuper de la salle. Celle-ci nous explique qu'elle ne fait pas de tri et que tous les dons sont exposés. Ce qui est rendu possible par le peu de dons faits : 30 à 40 par an. Cette muséographie de la totalité serait difficile à mettre en place à Trindade et à San Juan, où l'afflux de milliers de dons oblige les autorités ecclésiastiques à mettre en place d'autres stratégies dans la gestion de l'ex-voto.

À Sainte-Anne d'Auray, ce n'est pas la multiplication des miracles qui doit impressionner le visiteur mais leur narration. Ainsi, les menus objets avalés par les enfants inquiètent parce qu'ils sont associés à un écrit expliquant le danger. Rappelons que la présence d'une narration du miracle accompagnant le don est aussi l'un des critères du prêtre de San Juan pour laisser les ex-voto dans la salle. L'écrit confère donc à l'objet son caractère votif, tandis que sa présentation muséographique tend à en faire un objet historique. Ainsi, l'exposition de l'ex-voto à Sainte-Anne d'Auray oscille entre une valeur probatoire et un intérêt muséal ancré dans une volonté d'historisation et de patrimonialisation.

Mais l'exposition de son don est parfois nécessaire pour le croyant lui-même, nous l'avons vu pour San Juan. À Sainte-Anne, d'aucuns viennent également vérifier que leur don est bien en vitrine prêtant à l'exposition des vertus « magiques $»^{7}$. L'exposition entre alors dans le rituel de la pratique dévotionnelle. L'antiquité accordait une importance particulière à ce moment de remise de l'ex-voto, la dedicatio, soulignant le transfert de propriété à l'œuvre dans l'offrande (Turlan, 1955 : 513-514). La pratique votive est bien préservée et assumée en lui donnant une valeur patrimoniale, qui justifie en quelque sorte sa présence dans un lieu de culte catholique ancien. Ainsi les ex-voto historiques exposés et leur rareté attestent de l'ancienneté du culte, de sa pérennité et de sa force. 


\section{Conclusion}

L'examen du fonctionnement actuel de ces trois salles votives révèle donc que pour chaque site, les autorités ecclésiastiques utilisent et transforment la fonction de l'ex-voto une fois qu'il a été offert au divin. Ainsi à San Juan, son exposition se fait de manière fragmentaire mais, lorsqu'il s'agit d'un objet extrait du quotidien, il retrouve sa fonction première puisqu'il est revendu en tant qu'objet utilitaire ; à Trindade il est au service d'une forme de propagande catholique qui l'expose de manière monumentale pour accentuer sa valeur probatoire du miracle opéré ; à Sainte-Anne d'Auray l'ex-voto devient un objet historique témoin de la dévotion populaire parce qu'il est intégré à une logique de patrimonialisation. Si chaque lieu est dominé par une tendance générale, en réalité l'exposition de l'ex-voto dans le sanctuaire est le résultat d'une association de différentes valeurs. L'ex-voto après le don oscille ainsi entre une exposition à valeur probatoire et une muséographie du miracle à valeur patrimoniale, qui met en valeur ses qualités esthétiques. L'analyse de ces salles votives révèle alors la position complexe et ambiguë de l'Église à l'égard de l'objet votif car dans les trois cas, si elle conserve sa dimension votive, elle fait aussi évoluer le statut de l'ex-voto en le mettant au service d'une logique autre que celle du don.

Au-delà de cette gestion, l'on remarque l'extraordinaire contemporanéité et vitalité du fait votif, qui perdure depuis l'Antiquité au mépris des dogmes et du temps. Ni fossilisé, ni poussiéreux, l'ex-voto emprunte des formes différentes mais à travers le même type d'objets pour continuer à communiquer avec le divin. Cet usage actuel nous permet dès lors de comprendre la logique qui sous-tend la pratique votive. Objet trivial ou de valeur, il désigne une démarche en plusieurs étapes : la demande d'aide au divin, l'aide reçue et le don de l'objet promis. Plus qu'un objet, l'ex-voto est avant tout l'accomplissement d'une promesse. C'est cette valeur qu'il revêt pour les croyants et, si certains sont plus sensibles à son don qu'à son exposition, d'autres prêtent à cette même exposition des vertus magiques supplémentaires. Ainsi, l'ex-voto promis entretient des liens variés et complexes avec sa propre exposition. 


\section{Références}

Arias P., Durand J., 2002. La enferma eterna. Mujer y exvoto en México. Siglos XIX y XX. Guadalajara, Universidad de Guadalajara, El Colegio de San Luis.

Bélard M., Vérier P., 1997. Los exvotos del Occidente de México, México, CEMCA.

Bautier A.-M., 1977. Typologie des ex-voto mentionnés dans des textes antérieurs à 1200, In Actes du 99e Congrès National des Sociétés Savantes. Besançon, 1974. La piété populaire au Moyen Âge. Philologie et histoire jusqu'à 1610 Paris, Bibliothèque Nationale [France] pp. 473, 237-82.

Boullet F., Boullet C., 1978. Ex-voto marins. Genève, Éditions Maritimes et d'Outre-Mer.

Cousin B., 1979. L'Ex-voto, document d'histoire, expression d'une société/The Ex-voto, Historical Document, Expression of a Society, in Archives de sciences sociales des religions, N. 48/1, 107-124

Crémoux F., 2001. Pèlerinages et miracles à Guadalupe au XVIe siècle. Madrid, Casa de Velázquez. Cândido da Silva G., 2016. Diálogos possíveis: a comunicação museológica e a folkcominicação no Santuário da Bomfim, In Ex-votos do Brasil. Arte e folkcomunicação. Salvador, Quarteto.

Gruzinski S., 1990. La Guerre des Images, Paris, Fayard.

Gutiérrez Gutiérrez J., 2016. Valgame la Virgen de San Juan, narracion de los primeros milagros de la Vírgen de San Juan 1634 a 1734. San Juan de los Lagos, édité par la Basilique.

Heinich N., 2009. Ex-votos et curés d'Ars : l'inventaire de la dévotion en série, in In Situ, 12, http://insitu.revues.org/6424.

Huchet P., 2005. La Grande Histoire de Sainte-Anne d'Auray, Paris, Pierre Téqui.

Lepoittevin A., 1998. La chambre des merveilles votive du sanctuaire mantouan de Santa Maria delle Grazie. Le rôle des franciscains au XVIe siècle, in Mélanges de l'École française de Rome Italie et Méditerranée modernes et contemporaines, 126-2

Luke Agraz E., 2012. Análisis de la evolución de los exvotos pictóricos como documentos visuales para describir « la otra historia » de México. Thèse pour le doctorat de la UNED, Madrid.

Nora P., 1986. Les lieux de mémoire. Paris, Gallimard.

Oliveira Alves de J. C., 2011. Semiologia dos ex-votos no Brasil : simbolismo e comunicação religiosa, IX Congresso Lusocom | UNIP - São Paulo - SP 4 a 6 de agosto.

Perrée C., 2007. L'œuvre comme væu, l'œuvre comme don, l'ex-voto dans l'art contemporain. Thèse pour le doctorat de Paris I Sorbonne.

Prêtre C., 2014. L'offrande dans les inventaires de Délos : objet rituel ou sacré ?, in Revue de 
l'histoire des religions 4 ,

http://rhr.revues.org/8314.

Reinato E. J., 2009. Imaginário religioso nos ex-votos e nos vitrais da Basílica de Trindade - GO, in História: Debates e Tendências - v. 9, n. 2, jul./dez., 314-331.

Sigal P-A, 1985. L'Homme et le miracle dans la France médiévale (XI'-XII siècles). Paris, Éditions du C.E.R.F.

Sigal, P.-A. « L'ex-voto au Moyen Âge dans les régions du nord-ouest de la Méditerranée (XII ${ }^{\mathrm{e}}$ XV' siècle ». Provence historique, Tome 3, Fasc. 131, 1983, pp. 13-31

Turlan J., 1955. L'obligation « ex voto », in Revue historique de droit français et étranger 33, 504536. 